\title{
Use of Micronized Purified Flavonoid Fraction Together with Rivaroxaban Improves Clinical and Ultrasound Outcomes in Femoropopliteal Venous Thrombosis: Results of a Pilot Clinical Trial
}

Kirill Lobastov · Ilya Schastlivtsev • Victor Barinov

Received: October 25, 2018 / Published online: December 11, 2018

(C) The Author(s) 2018

\section{ABSTRACT}

Introduction: The aim of this study was to assess the impact of adding long-term micronized purified flavonoid fraction (MPFF) to standard treatment of femoropopliteal deep vein thrombosis (DVT).

Methods: This pilot, comparative, open-label study with blinded outcome assessor enrolled patients with a first episode of femoropopliteal DVT confirmed by duplex ultrasound scanning (DUS). All participants were randomly allocated to one of two treatment groups: (1) control that received a standard treatment with oral rivaroxaban, and (2) experimental that involved additional treatment with MPFF $1000 \mathrm{mg} /$ day. Both drugs were used for 6 months. Patients were followed for the whole treatment period and underwent DUS every 2 months to determine the degree of recanalization by popliteal $(\mathrm{PV})$, femoral vein (FV), and common femoral vein (CFV) compressibility. Thrombi extension were assessed by the modified Marder score. At the end of the follow-up period, patients were assessed with Villalta and venous clinical

Enhanced digital features To view enhanced digital features for this article go to https://doi.org/10.6084/ m9.figshare.7378130.

K. Lobastov ( $₫)$ · I. Schastlivtsev · V. Barinov Pirogov Russian National Research Medical

University, Moscow, Russian Federation

e-mail: lobastov_kv@hotmail.com severity scales (VCSS). Patients with a Villalta score $\geq 5$ were diagnosed with post-thrombotic syndrome (PTS).

Results: Sixty patients were randomized to the control or MPFF groups ( $n=30$ in each group). There were 40 men and 20 women with a mean age \pm SD of $56.3 \pm 13.4$ years. Clinically unprovoked DVT was recognized in 65\% of cases and left side localization in $45 \%$. The mean baseline Marder score was $15.0 \pm 4.8$ and $11.1 \pm 4.3$ in the experimental and control groups, respectively $(p=0.002)$. At 6 months, the mean Villalta score in the MPFF group was significantly lower compared with control $(2.9 \pm 2.7$ versus $5.8 \pm 3.0 ; p<0.0001)$. PTS was diagnosed in six patients (20\%) and 17 patients (57\%) in the experimental and control groups respectively $(p=0.007)$. A significant difference between the groups was also observed for the VCSS value $(2.3 \pm 1.9$ versus $4.9 \pm 1.9$; $p<0001)$. After 6 months of treatment the Marder score decreased to $0.8 \pm 1.6$ and $2.8 \pm 3.5$ in the experimental and control groups, respectively $(p=0.006)$. In the MPFF group, there was a greater reduction in the Marder score $(p<0.0001)$ and more rapid rate of recanalization for the FV $(p<0.0001)$, with a non-significant trend for the CFV $(p=0.130)$ and PV $(p=0.204)$ compared with the control group. Full recanalization of the PV at 6 months was observed in 24 patients $(80 \%)$ who had received MPFF, and only 17 patients (57\%) in the control group $(p=0.095)$. 
Conclusion: The addition of MPFF to standard therapy for DVT in the form of oral rivaroxaban can reduce the incidence of PTS at 6 months in patients with proximal DVT and increase the speed of deep vein recanalization.

Funding: Les Laboratoires Servier funded the article processing fees, editorial assistance, and open access fee for this manuscript.

Keywords: Cardiology; Deep vein thrombosis; Micronized purified flavonoid fraction; Postthrombotic syndrome; Recanalization; Rivaroxaban

\section{INTRODUCTION}

Deep vein thrombosis (DVT) is a significant medical and social problem. According to official statistics, the incidence of DVT in the Russian Federation remained stable throughout the period 2012-2016 accounting for 1.5-1.6 cases per 1000 population per year [1-3], which is slightly higher than figures obtained in published epidemiological studies [4-8]. The main long-term issue after an episode of DVT is the occurrence of post-thrombotic syndrome (PTS), which significantly affects the patients' quality of life. The incidence of PTS in the 10-15 years after the primary thrombotic episode reaches $19-42 \%$, and severe forms of the disease with the presence of trophic disorders can be detected in 3-4\% of patients [9-12]. The symptoms of PTS usually develop within the first 6 months following the primary DVT, but can occur years after the initial event [9].

The pathogenetic mechanisms leading to the development of PTS may involve lesions of the deep vein valves with the formation of blood reflux, incomplete recanalization of venous segments with the persistence of residual venous obstruction, or a combination of both mechanisms [13]. The most important component is the presence of residual venous obstruction, which increases the risk of developing PTS by 1.6-2.0-fold [14-18]. The recanalization of veins is a complex biological process. One of its integral components is the immune inflammatory reaction, which is responsible for clearance of thrombotic masses from the lumen of the vessel and venous wall lesion $[19,20]$. Previous studies have shown that increased levels of inflammatory markers, in particular C-reactive protein, interleukins-1 and -6 , intercellular adhesion molecule-1 (ICAM-1), and a reduced level of anti-inflammatory cytokines (interleukin-10) are associated with a higher risk of PTS development [21-25]. This raises the possibility that pharmacological modulation of the inflammatory reaction may protect the venous wall from excessive leukocyte aggression, while retaining the natural role of immune cells in the process of vessel recanalization.

Among the potential pharmacological agents with the ability to target the inflammatory reaction directly in the venous wall, micronized purified flavonoid fraction (MPFF), an oral agent, is of particular interest. Previous experimental studies have demonstrated its prominent endothelial protective properties in the form of reduced rolling, adhesion, and migration of leukocytes in the settings of reperfusion injury and secondary venous hypertension [26-29]. It has also been shown to suppress the inflammatory reaction in patients with chronic venous disease (CVD) [30], including those who have undergone sclerotherapy [31]. To test the hypothesis that long-term treatment with MPFF may affect the natural course of DVT, this study evaluated the impact of MPFF use in the treatment of patients with femoropopliteal DVT.

\section{METHODS}

This pilot, single-center, open-label comparative clinical trial with blinded outcome assessment recruited patients over 18 years of age with a first episode of clinically provoked or unprovoked DVT in the femoropopliteal vascular segment with possible involvement of the calf veins and the common femoral vein. DVT location was confirmed by duplex ultrasound scan (DUS). All procedures performed in studies involving human participants were in accordance with local ethics committee of the Clinical Hospital No. 1 of the Presidents Administration of Russian Federation and with the 1964 Helsinki declaration and its later 
amendments or comparable ethical standards. Informed consent was obtained from all individual participants included in the study. At the authors' institution it is not obligatory to register pilot and unfunded studies; therefore this trial is not registered.

Exclusion criteria were suspected or confirmed pulmonary embolism; bilateral DVT location; extension of the thrombosis into the iliac veins above the inguinal fold; contraindications to therapy with anticoagulants or MPFF; verified malignant tumor at the time of DVT manifestation; known severe thrombophilia (deficiency of protein $\mathrm{C}$, protein $\mathrm{S}$, antithrombin III, antiphospholipid syndrome); use of parenteral anticoagulants for 7 days or more from the time of DVT verification; inability to use compression stockings; any intervention for DVT (inferior vena cava filter implantation, thrombectomy, or catheter-guided thrombolysis); continued use of other drugs affecting the hemostatic system (except acetylsalicylic acid at a dose of up to $100 \mathrm{mg}$ per day); and poor compliance.

The study was carried out at the facilities of the Clinical Hospital No. 1 of the Presidents Administration of the Russian Federation in 2017-2018. In accordance with the internal protocol of the institution, all patients with suspected DVT entering the emergency department were assessed using a two-level Wells score and a D-dimer evaluation [32]. In patients with a high clinical probability of DVT or a combination of low clinical probability for DVT with an increased level of D-dimer, anticoagulant therapy was initiated with low molecular weight heparin (LMWH), followed by DUS. Patients with diagnosed femoropopliteal venous thrombosis were assessed for fulfillment of the inclusion and exclusion criteria. After signing informed consent, patients were randomly allocated to one of two groups based on the number on the patient's medical record form: those whose last digit was even were assigned to the MPFF group, and those whose last digit was odd were assigned to the control group; if the last digit was zero, the penultimate digit was used. In the case of premature completion of recruitment to either group, all eligible patients were intentionally allocated to the second group.

During the period from hospital admission to treatment allocation, all patients received therapeutic doses of LMWH (enoxaparin $1 \mathrm{mg}$ / $\mathrm{kg}$ twice daily). After randomization, patients in both groups were switched to the oral anticoagulant rivaroxaban at a dosage of $15 \mathrm{mg}$ twice daily for up to 3 weeks from the administration of the first dose of LMWH. Thereafter they received a dosage of $20 \mathrm{mg}$ once daily for up to 6 months. The first dose of rivaroxaban was administered instead of LMWH at the time of the next scheduled injection. In addition, during the first 3 days following the DVT diagnosis, patients in both groups applied individualized medical compression in the form of thigh-high stockings with a pressure of $23-32 \mathrm{mmHg}$ (class 2 compression according to RAL-GZ 387 standard). Matching the size of the stocking to the diameter of the limb was controlled 3 weeks and 3 months after the start of treatment. Patients were advised to wear the stockings $24 \mathrm{~h}$ a day for the first week and then during the day for the next 6 months. In the MPFF group, patients received MPFF $500 \mathrm{mg}$ twice daily for 6 months in addition to standard treatment. The first dose of MPFF was administered immediately after treatment allocation and in parallel with the switch to the oral form of the anticoagulant.

Patients were followed for 6 months with clinical and ultrasound evaluation every 2 months. At baseline, in addition to standard clinical data, the affected lower limb was evaluated for the presence of pre-existing CVD, and if confirmed, the clinical class according to the CEAP (Clinical, Etiological, Anatomical, and Pathological) classification was determined. CEAP clinical classes C1 (telangiectases), C2 (varicose veins), C4 (skin pigmentation, eczema, lipodermatosclerosis or athrophie blanche), C5 (healed venous ulcer), and C6 (active venous ulcer) were confirmed objectively on the basis of the presence of corresponding signs of the disease. CEAP clinical class C3 (presence of leg edema) was confirmed on the basis of the patient's medical history (complaints of edema of the lower limb or need for compression therapy prior to the manifestation of thrombosis), as at the time of physical 
examination all patients had swelling due to acute deep vein occlusion. CEAP class was also determined after 6 months of treatment. CVD severity was assessed using the Venous Clinical Severity Score (VCSS) [33]. The VCSS includes 10 clinical descriptors (pain, varicose veins, venous edema, skin pigmentation, inflammation, induration, number of active ulcers, duration of active ulceration, size of ulcer, and compressive therapy use), scored from 0 to 3 (total possible score, 30). Quality of life was assessed using the Chronic Venous Disease quality of life Questionnaire (CIVIQ-20), a validated, 20-item, self-reported scale that captures the key dimensions of quality of life specifically impaired by CVD in the following four domains: physical, psychological, social, and pain [34]. The presence and severity of PTS was assessed using the Villalta score, which is based on the sum of severity ratings of five venous symptoms and six clinical signs (Table 1) [35]. The final judgement about the presence of PTS and the severity of CVD was made by an independent expert who was blinded to the patient's allocation to MPFF or control group.

DUS was performed using the MyLab30 device (Esaote, Italy) with a linear ultrasound transducer LA532 in the frequency range 5-13 MHz. The common femoral vein (CFV) and femoral vein (FV) were assessed in the supine position, popliteal vein (PV) in the prone position, and calf veins in a sitting position. At each examination, all accessible venous segments were subject to evaluation.

The criteria for DVT were incompressibility of the vein during compression by the transducer and absence of blood flow in the vein on the color mapping mode. To assess the prevalence of thrombotic occlusion, a modified Marder score was used. This allocates points to each of the deep and superficial venous segments of the lower limb that are occluded. If all the veins of one leg are occluded the total score is 34 (Table 2) [36]. When performing the dynamic DUS, the degree of recanalization of the $\mathrm{CFV}, \mathrm{FV}$, and PV was calculated on the basis of their compressibility. This was achieved by

Table 1 Clinical criteria for post-thrombotic syndrome (Villalta score [13])

\begin{tabular}{lllll}
\hline & None & Mild & Moderate & Severe \\
\hline 5 symptoms & & & & \\
Pain & 0 points & 1 point & 2 points & 3 points \\
Cramps & 0 points & 1 point & 2 points & 3 points \\
Heaviness & 0 points & 1 point & 2 points & 3 points \\
Paresthesias & 0 points & 1 point & 2 points & 3 points \\
Pruritus & 0 points & 1 point & 2 points & 3 points \\
6 clinical signs & & & & \\
Pretibial edema & 0 points & 1 point & 2 points & 3 points \\
Hyperpigmentation & 0 points & 1 point & 2 points & 3 points \\
Venous ectasia (venules or varicose veins) & 0 points & 1 point & 2 points & 3 points \\
Redness & 0 points & 1 point & 2 points & 3 points \\
Skin induration & 0 points & 1 point & 2 points & 3 points \\
Pain on calf compression & 0 points & 1 point & 2 points & 3 points \\
\hline
\end{tabular}

Total score of 0-4 indicates no PTS; score of $\geq 5$ indicates PTS. PTS severity: total score of 5-9, mild PTS; score of 10-14, moderate PTS; and score of $\geq 15$ or venous ulcer present, severe PTS 
Table 2 Ultrasound quantification of deep vein thrombosis (modified Marder score [36])

\begin{tabular}{ll}
\hline Venous segment examined & Score \\
\hline Iliac vein & 8 \\
Common femoral vein & 4 \\
Femoral vein & 4 \\
Deep femoral vein & 2 \\
Popliteal vein & 2 \\
Tibial trunk & 2 \\
Peroneal trunk & 2 \\
Tibial vein (both of the pair) & $1(2)$ \\
Peroneal vein (both of the pair) & $1(2)$ \\
Gastrocnemius vein (both medial and lateral) & $1(2)$ \\
Soleal vein or other calf muscle vein & 1 \\
Great saphenous vein trunk & 2 \\
Small saphenous vein trunk & 1 \\
Maximal score for one limb & 34 \\
\hline
\end{tabular}

measuring the veins' diameters without compression and at maximum compression with the transducer at the narrowest point of each venous segment. The degree of recanalization was calculated with the following formula: [ $d$ (no compression) $-d$ (maximal compression) $] / d$ (no compression) $\times 100 \%$. All measurements were repeated three times, and an average value was used for calculations. All ultrasound studies were performed by a specialist blinded to the patient's allocation to the MPFF or control group.

The primary study endpoint was the presence of PTS signs at 6 months, determined by a blinded expert. For the diagnosis of PTS, the Villalta score was used. The criteria for a PTS diagnosis were a Villalta score of $\geq 5$; severe PTS was defined as a score of $\geq 15$ or the presence of an active venous ulcer.

The secondary study endpoints included severe PTS; symptomatic or asymptomatic recurrence of DVT; symptomatic pulmonary embolism; change in CEAP clinical class; CVD severity assessed by VCSS, quality of life assessed by CIVIQ-20; complete recanalization of vessels; change in degree of recanalization for the CFV, $\mathrm{FV}$, and PV; change in thrombus extension by the Marder score; and the occurrence of bleeding and/or other adverse events associated with therapy.

Symptomatic recurrence of DVT was defined as an increase in edema, pain, or hyperemia of the affected lower limb, or the occurrence of the same signs on the intact leg. The asymptomatic recurrence of DVT was defined as the occurrence of signs of total occlusion in a previously recanalized venous segment. A complete recanalization was the clearance of thrombotic masses by $80 \%$ or more in the venous lumen (residual vein occlusion less than 20\%).

Bleeding related to anticoagulant therapy was classified as major (according to the International Society on Thrombosis and Hemostasis criteria) [37], clinically relevant non-major (any episode not fulfilling the criteria of major bleeding, but resulting in withdrawal of anticoagulant therapy and/or requiring a specific medical intervention and/or leading to an unscheduled visit to the doctor), or minor (any episode not fulfilling the criteria of major or clinically relevant non-major bleeding).

Patients' general health status was assessed at each follow-up visit with an emphasis on identifying possible adverse events of therapy.

\section{Statistical Methods}

As this was a pilot study with an exploratory nature the minimum sample size was not calculated. Statistical analysis was carried out using IBM SPSS Statistics v.19 software package. All absolute values are presented as the mean value with the standard deviation (mean \pm SD). The distribution was verified by a Kolmogorov-Smirnov test. If a normal distribution was confirmed, comparisons were performed using the $t$ test for independent samples for continuous variables, or the two-sided Fisher's exact test and a Chi-square test for categorical variables. Comparisons of mean values with time were performed by assessment of withinand between-subject effects, as well as their 
within-subject interaction using the general linear model for repeated measurements (GLM$\mathrm{RM})$. Differences were considered statistically significant when the $p$ value was less than 0.05 .

\section{RESULTS}

During the inclusion period, 132 patients with suspected DVT were admitted to the hospital and thrombosis was confirmed in 104 cases. Of these, 68 patients fulfilled the selection criteria and 8 patients refused to participate. The remaining 60 patients were allocated to one of the two treatment groups $(n=30$ in each group), and all patients completed the study (Fig. 1).

The clinical characteristics of the groups are presented in Table 3. Patients in the control group receiving standard treatment had a higher rate of CVD prior to the occurrence of DVT, in particular CEAP clinical class C2-C4.
Patients in the two groups were comparable in terms of the prevalence of lesions of the main vessels in the femoropopliteal venous segment, although the total extent of the pathological process as determined by the Marder score was higher in the MPFF group (Table 3). These differences were associated primarily with the greater involvement of calf veins in patients treated with MPFF.

The results for the primary and secondary endpoints are summarized in Table 4. PTS was reported in $56.7 \%$ patients after 6 months of anticoagulant therapy with rivaroxaban and compression therapy and in $20 \%$ of patients randomized to MPFF plus standard therapy $(p=0.007)$. No severe forms of PTS were reported in either group. The mean score on the Villalta scale and the mean VCSS value were also lower in patients in the MPFF group. The CIVIQ-20 global index score revealed an improved quality of life in the MPFF group.

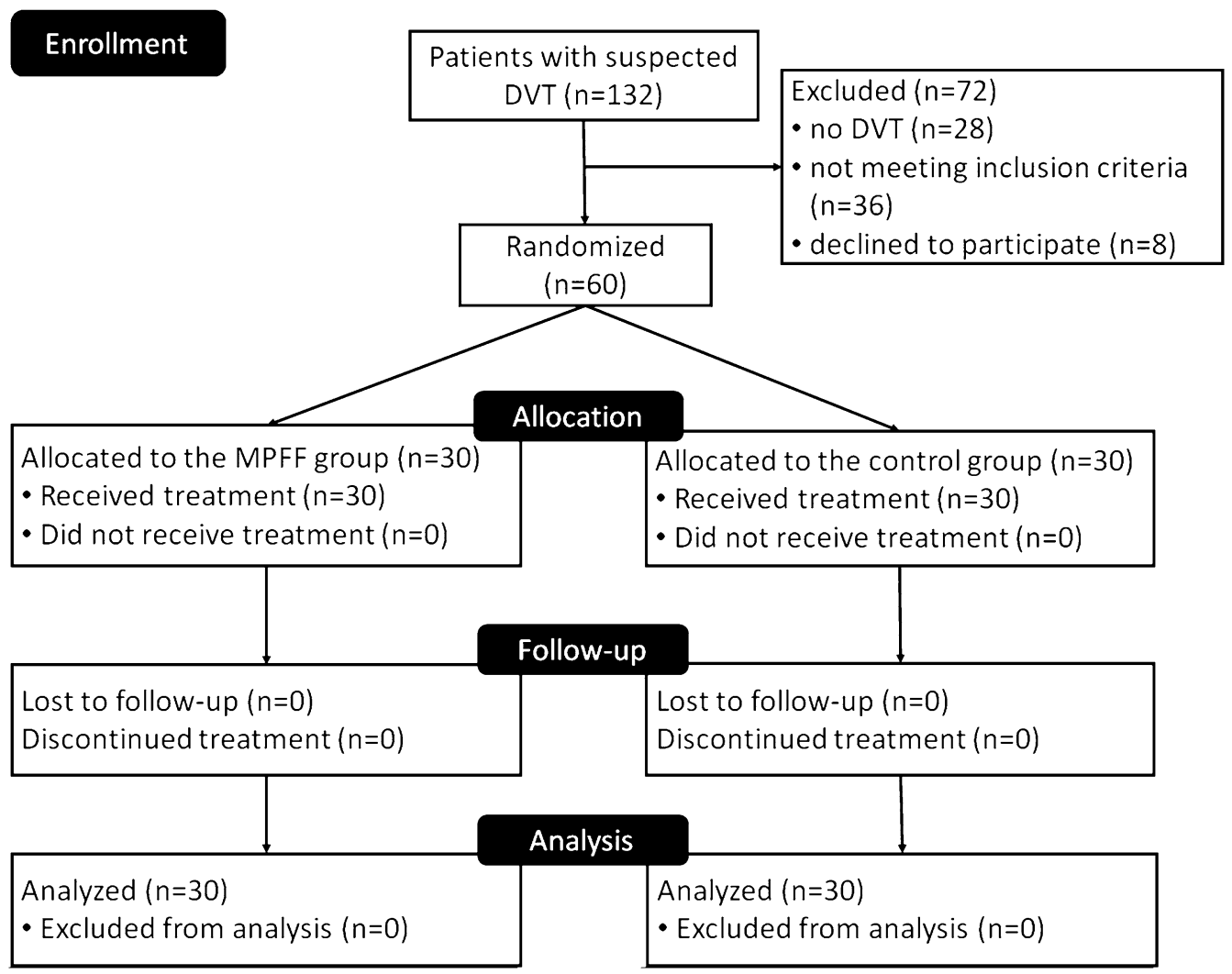

Fig. 1 CONSORT flowchart of the study 
Table 3 Characteristics of patients in the MPFF and control groups

\begin{tabular}{llll}
\hline Parameter & MPFF $(\boldsymbol{n}=\mathbf{3 0})$ & Control $(\boldsymbol{n}=\mathbf{3 0})$ & $\boldsymbol{p}$ value \\
\hline Age (years, mean \pm SD) & $55.2 \pm 14.9$ & $57.5 \pm 11.9$ & 0.518 \\
Male (\%) & 56.7 & 76.7 & 0.170 \\
Clinically unprovoked DVT (\%) & 56.7 & 73.3 & 0.279 \\
Duration of symptoms by time of hospital admission (days, mean \pm SD) & $4.3 \pm 3.7$ & $4.2 \pm 2.6$ & 0.936 \\
Time from hospital admission to allocation (days, mean \pm SD) & $3.9 \pm 1.2$ & $3.9 \pm 1.7$ & 0.931 \\
History of CVD (\%) & 50.0 & 73.4 & 0.110 \\
Class C0 & 23.3 & 23.3 & 0.014 \\
Class C1 & 26.7 & 3.3 & \\
Class C2 & 33.3 & 46.8 & \\
Class C3 & 16.7 & 13.3 & \\
Class C4 & 0.0 & 13.3 & \\
Lesions of the left lower extremity (\%) & 50.0 & 40.0 & 0.604 \\
Thrombus extension by Marder score (mean \pm SD) & $15.0 \pm 4.8$ & $11.1 \pm 4.3$ & 0.002 \\
CFV lesion (\%) & 43.3 & 33.3 & 0.596 \\
FV lesion (\%) & 83.3 & 66.7 & 0.233 \\
PV lesion (\%) & 100.0 & 100.0 & 1.000 \\
\hline
\end{tabular}

$D V T$ deep vein thrombosis, $S D$ standard deviation, $C V D$ chronic venous disease, $C F V$ common femoral vein, $F V$ femoral vein, $P V$ popliteal vein

Progression of pre-existing CVD was observed in one patient in the MPFF group (transition from $\mathrm{C} 2$ to $\mathrm{C} 4$ class) and six patients in the control group (transition from $\mathrm{C} 2$ to $\mathrm{C} 4$ class in one patient, and transition from $\mathrm{C} 2$ to C3 class in five patients). Thus, the signs of CVD progression were observed in $20 \%$ of patients receiving standard therapy for DVT and only in $3.3 \%$ of patients with additional use of MPFF.

The recurrence of a venous thromboembolic event was observed in one patient from the control group, who had a symptomatic recurrent DVT on the contralateral limb, which required a temporary switch to LMWH followed by therapy with vitamin $\mathrm{K}$ antagonists. No major bleedings were observed in either group. Clinically relevant, non-major bleedings were represented by one case of hemorrhoidal bleeding in the MPFF group and two cases of macrohematuria in the control group. In addition, two cases of minor bleeding were reported (one in each group): rectal bleeding in the MPFF group and epistaxis in the control group.

In terms of other adverse effects, three patients in the MPFF group experienced a mild dyspeptic disorder, which did not require treatment discontinuation.

Rates of recanalization in the main venous segments are presented in Fig. 2. In both groups, there was a significant trend towards progressive clearance of thrombotic masses from the vessel lumen $(p<0.0001$ for withinsubject effect "time"). There were no significant differences in the rate of recanalization in the popliteal vein between the two groups, and complete recovery of vascular patency was achieved in $80 \%$ of patients in the MPFF group and $57 \%$ of patients in the control group 
Table 4 Rates of the primary and secondary endpoints in the MPFF and control groups

\begin{tabular}{llll}
\hline Endpoint & MPFF $(\boldsymbol{n}=30)$ & Control $(\boldsymbol{n}=30)$ & $\boldsymbol{p}$ value \\
\hline Primary & & $17(56.7 \%)$ & 0.007 \\
Diagnosis of PTS & $6(20.0 \%)$ & & - \\
Secondary & & 0 & $<0.0001$ \\
Diagnosis of severe PTS & 0 & $5.8 \pm 3.0$ & $<0.0001$ \\
Villalta score, mean \pm SD & $2.9 \pm 2.7$ & $4.9 \pm 1.9$ & $<0.0001$ \\
VCSS value, mean \pm SD & $2.3 \pm 1.9$ & $31.6 \pm 8.5$ & 0.057 \\
CIVIQ-20 score, mean \pm SD & $24.1 \pm 4.6$ & $6(20.0 \%)$ & 1.000 \\
Progression in CEAP clinical class & $1(3.3 \%)$ & $1(3.3 \%)$ & 1.000 \\
Recurrence of VTE & 0 & $3(10.0 \%)$ & - \\
Bleeding & $2(6.7 \%)$ & 0 & 1.000 \\
Major & 0 & $2(6.7 \%)$ & 1.000 \\
Clinically relevant non-major & $1(3.3 \%)$ & $1(3.3 \%)$ & 0
\end{tabular}

SD standard deviation, PTS post-thrombotic syndrome, CEAP Clinical, Etiological, Anatomical, and Pathological classification, CIVIQ-20 Chronic Venous Disease quality of life Questionnaire, VCSS Venous Clinical Severity Score, VTE venous thromboembolic event

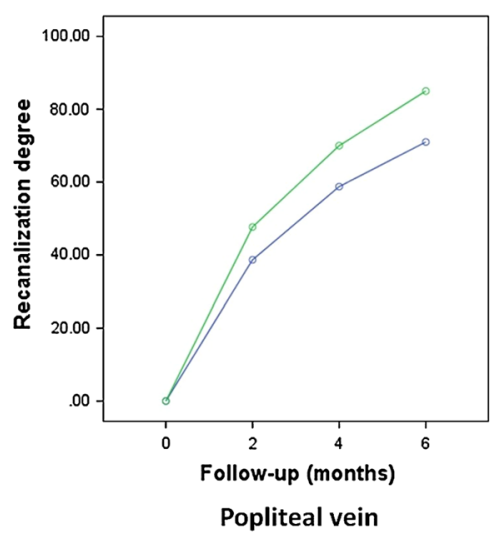

$\mathrm{p} 1<0.0001, \mathrm{p} 2=0.204, \mathrm{p} 3=0.107$

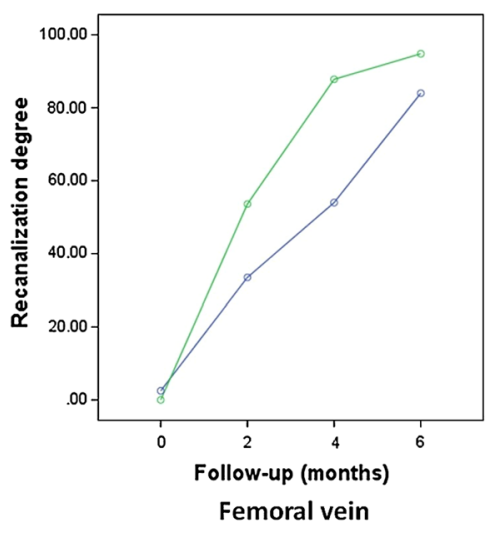

$\mathrm{p} 1<0.0001, \mathrm{p} 2<0.0001, \mathrm{p} 3=0.001$

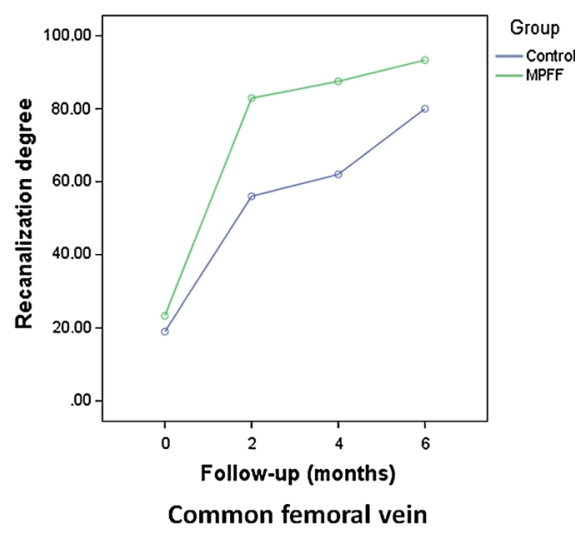

$\mathrm{p} 1<0.0001, \mathrm{p} 2=0.130, \mathrm{p} 3=0.143$

Fig. 2 Rates of recanalization for the main venous segments. Generalized linear model repeated measures: p1 within-subject effect "time", p2 within-subject interaction "time $\times$ group", p3 between-subject effect "group"

$(p=0.095)$. The recanalization of the femoral vein, in turn, occurred significantly faster in patients receiving MPFF ( $p<0.0001$ for withinsubject interaction "time $\times$ group"); however, the rate of achieving complete recovery of vascular patency was not significantly different: $92 \%$ in the MPFF group vs. $75 \%$ in the control group $(p=0.214)$. 


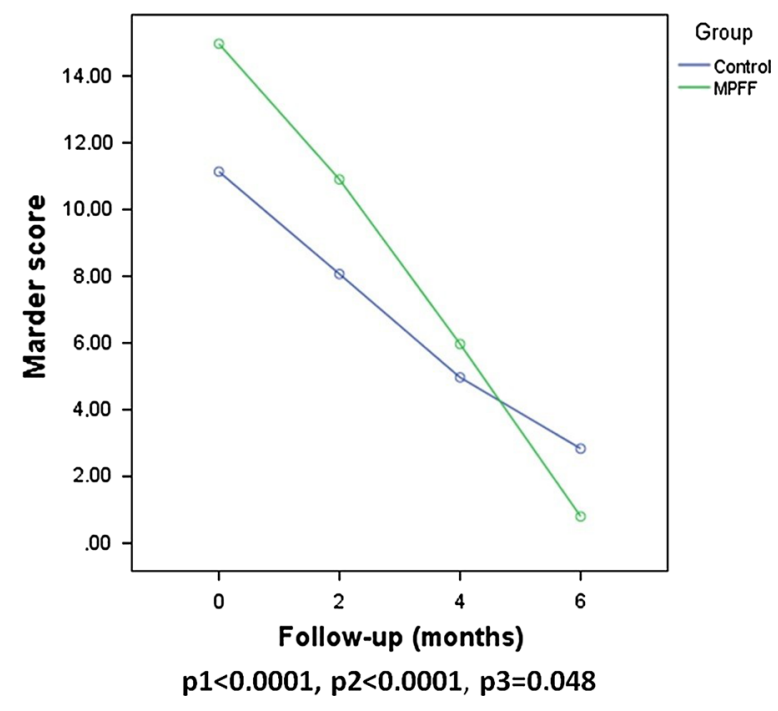

Fig. 3 Changes in the Marder score. Generalized linear model repeated measures: p1 within-subject effect "time", p2 within-subject interaction "time $\times$ group", p3 betweensubject effect "group"

There were no significant differences between the groups in the CFV analyses, both in terms of the rate of recanalization and in the rate of achieving complete recovery: $92 \%$ in the MPFF group vs. $70 \%$ in the control group $(p=0.293)$. At the same time, significant differences between the groups in total extent of the thrombus were revealed using the Marder score (Fig. 3). At baseline, the extent of thrombus in the MPFF group was significantly higher than in the control group $(15.0 \pm 4.8$ vs. $11.1 \pm 4.3$, respectively; $p=0.002$ ), but after 6 months of therapy it was significantly lower $(0.8 \pm 1.6$ vs. $2.8 \pm 3.5$ points, $p=0.006)$. This suggests that long-term use of MPFF in addition to standard DVT therapy provides a faster reduction in thrombus burden.

\section{DISCUSSION}

Recanalization of a thrombosed vein is a complex biological process, an integral component of which is the immune inflammatory reaction involving a large number of neutrophils, monocytes, and lymphocytes $[19,20]$. The clearance of thrombus from the vessel lumen involves lysis of the fresh clot triggered by activation of the plasma fibrinolytic system, fragmentation of the thrombotic masses by endothelial lytic activity, contraction and retraction of the thrombus as a result of active formation of collagen on the framework of fibrin filaments, and also through the formation of new channels, lined with endothelium, directly in the thrombotic mass [38]. At the same time, thrombus resolution is strongly correlated with preservation of valvular function of the affected vessels [39]. However, initial attempts to treat DVT with unfractionated heparin followed by a switch to long-term intake of vitamin $\mathrm{K}$ antagonists led to inconsistent results in terms of recanalization of the affected venous segments. In approximately $50 \%$ of cases, there were no changes or an increase in the extent of thrombosis after 5-10 days of treatment, and after 3-6 months the number of patients with an unchanged thrombus burden reached 23\% [40].

At the same time, the long-term use of LMWH in comparison with standard therapy with vitamin $\mathrm{K}$ antagonists increased the chance of complete vascular recanalization 1.5fold and reduced the probability of PTS occurrence by $23 \%$ [41]. Most likely, this was due to the pronounced pleiotropic effects of LMWH and the presence of anti-inflammatory, profibrinolytic, antisclerotic, and endothelioprotective properties in addition to anticoagulant activity [42].

The introduction of direct oral anticoagulants was a milestone in DVT therapy, simplifying the treatment regimen, improving safety while maintaining high efficiency, and also eliminating the need for routine laboratory monitoring of treatment adequacy [43]. In addition, it has been shown that treating venous thrombosis with rivaroxaban is associated with a higher rate of recanalization of venous segments and a lower risk of PTS [44-46]. The exact mechanisms of action have not been fully elucidated, but it is assumed that direct oral anticoagulants, and in particular rivaroxaban, have certain anti-inflammatory properties, which can be mediated through protease-activated receptors [47, 48]. However, this hypothesis requires experimental confirmation. 
When assessing the effect of anticoagulant therapy on the completeness of recanalization of thrombosed vessels, a particular difficulty is the interpretation of the clinical significance of residual venous obstruction. Thus, Piovella et al. and Siragusa et al. consider residual thrombus as "clinically significant" if it occupies more than $40 \%$ of the vessel lumen $[49,50]$. At the same time, Prandoni et al. in their early studies defined residual obstruction in the femoropopliteal segment to be significant if vein thickness at maximum compression exceeded 2-3 mm [51, 52]. In more recent studies evaluating the degree of vascular recanalization in patients receiving direct oral anticoagulants, the cutoff for vein thickness was increased to $4 \mathrm{~mm}$ [46]. Given this uncertainty, the rate of residual venous obstruction detection, as well as its impact on disease outcome, can vary substantially from study to study. In this study, we assumed a clinically significant residual obstruction at $20 \%$ of the vessel cross-sectional diameter, which is approximately $1-2 \mathrm{~mm}$ for the femoropopliteal segment and corresponds to the early criteria of Prandoni et al. [51, 52]. When this cutoff value was used, the rate of residual venous obstruction diagnosis on rivaroxaban monotherapy was $43 \%$ for the popliteal vein, $25 \%$ for the femoral vein, and $30 \%$ for the common femoral vein. These figures are significantly higher than that obtained using a 4-mm cutoff value, at which the rate of residual obstruction diagnosis was only $21.1 \%$ [46].

When long-term use of rivaroxaban was combined with MPFF, the rate of residual venous obstruction was reduced to $20 \%$ for the popliteal vein, and $8 \%$ for the femoral vein and common femoral vein, which indicates a significant improvement in the recanalization process with the addition of flavonoid. The benefits of MPFF were even more pronounced when analyzed by changes in the Marder score reflecting the total extent of thrombus.

The addition of MPFF resulted in a more rapid and complete clearance of thrombotic masses from the blood vessels, and as a result, by the end of the 6-month follow-up period the scores in the MPFF group were significantly lower than in the control group. The observed differences can be explained by the known antiinflammatory activity of MPFF. Previous research in patients with CVD has shown that MPFF treatment for 60 days decreased levels of cellular adhesion molecules (ICAM- 1 by $32 \%$ and VCAM by 29\%) in the regional venous blood [30]. In women with CVD class CEAP C1, significant increases in levels of C-reactive protein, histamine, interleukin-1, tumor necrosis factor-alpha, and vascular endothelial growth factor have been observed in blood samples taken from the vessel supplying the telangiectasia 10 days after microsclerotherapy [31]. When sclerotherapy is performed with adjunctive MPFF the activity of these inflammatory markers is significantly reduced. Further studies are now warranted to confirm whether MPFF can also affect levels of inflammatory biomarkers in venous thrombosis.

A further difficulty when assessing long-term DVT outcomes is objective confirmation of the presence of PTS. In previous studies, the rate of PTS diagnosis by the Villalta scale in patients treated with rivaroxaban was $25-45 \%$ for a mean follow-up period of about 2 years [44, 45]. In our study, the rate of PTS diagnosis after 6 months of rivaroxaban monotherapy was higher at $56.7 \%$. This may be due to the peculiarities of the Villalta scale, which has a high sensitivity to the presence of pre-existing CVD. It is known that the presence of primary venous insufficiency prior to the development of DVT raises the risk of PTS occurrence 1.5-3.2-fold [13]. Moreover, the Villalta scale has been reported to result in a misdiagnosis of PTS in $42 \%$ of patients who do not have specific postthrombotic changes in the deep veins [53]. The high sensitivity of this tool to pre-existing CVD can result in a wide dispersion in the rates of registered long-term adverse DVT outcomes. In our study, about three-quarters of the patients in the control group had a primary lesion of the superficial veins before the development of thrombosis, which could have had an impact on the high prevalence of PTS. At the same time, around half the patients in the MPFF group also had pre-existing CVD. However, signs of disease progression in terms of a worsening in CEAP clinical class to more severe forms were much less common with the use of 
the MPFF (3.3\% vs. 20\%). This apparent trend may be associated with the ability of MPFF to reverse the symptoms and signs of CVD, and thus have a positive effect on the prevention and treatment of early forms of PTS [54].

The current study has demonstrated the benefits of using MPFF together with rivaroxaban for the long-term treatment of DVT. The addition of MPFF not only allowed the rate of PTS to be reduced, but also accelerated recanalization of affected venous segments. At the same time, the proposed therapeutic approach was associated with a high safety profile; no serious events associated with longterm intake of MPFF were recorded, and all the identified adverse events were mild in nature and did not result in treatment discontinuation.

This preliminary research had a pilot study design and was subject to certain limitations. First, the small sample size did not allow adjustments to be made for differences between treatment groups, in particular the extent of thrombus and the presence of pre-existing CVD. When planning further studies, appropriate randomization methods should be used to eliminate any source of bias in treatment assignments, patient recruitment should be carefully balanced for pre-existing CVD or exclude participants with this condition. Other important limitations were the absence of a placebo control and the short follow-up period. These limitations must be overcome in subsequent studies. Larger randomized controlled trials are now required to establish the superiority of the combined regimen before routine use in clinical practice can be recommended.

\section{CONCLUSION}

Preliminary data demonstrate that long-term therapy for DVT with a combination of rivaroxaban and MPFF reduced the rate of PTS development compared with rivaroxaban monotherapy and accelerated recanalization of affected venous segments. These results now need to be confirmed in larger randomized, double-blind, placebo-controlled studies.

\section{ACKNOWLEDGEMENTS}

We thank the participants of the study. The study was carried out at the facilities of the Clinical Hospital No. 1 of the Presidents Administration of the Russian Federation in 2017-2018.

Funding. The open access fee, article processing charges, and editorial assistance for the production of this article were funded by Les Laboratoires Servier. The study was conducted without any financial or technical support. All authors had full access to all of the data in this study, contributed to the concept and interpretation of the literature, were involved in the drafting and revising of the paper and approval of the final submitted version, and take complete responsibility for the integrity of the data and accuracy of the data analysis.

Editorial Assistance. Editorial assistance for English language editing was provided by Jenny Grice, Bieuzy les Eaux, France and funded by Les Laboratoires Servier.

Authorship. All named authors meet the International Committee of Medical Journal Editors (ICMJE) criteria for authorship for this article, take responsibility for the integrity of the work as a whole, and have given their approval for this version to be published.

Disclosures. Kirill Lobastov, Ilya Schastlivtsev and Victor Barinov have nothing to disclose.

Compliance with Ethics Guidelines. All procedures performed in studies involving human participants were in accordance with local ethics committee of the Clinical Hospital No. 1 of the Presidents Administration of Russian Federation and with the 1964 Helsinki declaration and its later amendments or comparable ethical standards. Informed consent was obtained from all individual participants included in the study.

Data Availability. The datasets generated and/or analyzed during the current study are 
available from the corresponding author on reasonable request.

Open Access. This article is distributed under the terms of the Creative Commons Attribution-NonCommercial 4.0 International License (http://creativecommons.org/licenses/ by-nc/4.0/), which permits any noncommercial use, distribution, and reproduction in any medium, provided you give appropriate credit to the original author(s) and the source, provide a link to the Creative Commons license, and indicate if changes were made.

\section{REFERENCES}

1. Общая заболеваемость всего населения России в 2012 году. Статистические материалы, Часть II 2013. [The general prevalences of diseases in the total population of Russia in 2012. Statistical Materials, Part II 2013 (In Russian)]. http://www.rosminzdrav. ru/documents/8029-statisticheskaya-informatsiya2012. Accessed 19 Oct 2018.

2. Заболеваемость всего населения России в 2014 году. Статистические материалы, Часть II 2015 [The prevalences of diseases in the total population of Russia in 2014. Statistical Materials, Part II 2015 (In Russian)]. https://www.rosminzdrav.ru/documents/ 9479-statisticheskaya-informatsiya-za-2014. Accessed 19 Oct 2018.

3. Заболеваемость всего населения России в 2016 году. Статистические материалы Часть II 2017. [The prevalences of diseases in the total population of Russia in 2016. Statistical Materials, Part II 2017 (In Russian)]. https://www.rosminzdrav.ru/ministry/ 61/22/stranitsa-979/statisticheskie-iinformatsionnye-materialy/statisticheskiy-sbornik2016-god. Accessed 19 Oct 2018.

4. Hippisley-Cox J, Coupland C. Development and validation of risk prediction algorithm (QThrombosis) to estimate future risk of venous thromboembolism: prospective cohort study. BMJ. 2011;343:d4656.

5. Oger E. Incidence of venous thromboembolism: a community-based study in Western France. EPIGETBP Study Group. Groupe d'Etude de la Thrombose de Bretagne Occidentale. J Thromb Haemost. 2000;83(5):657-60.

6. Naess IA, Christiansen SC, Romundstad P, Cannegieter SC, Rosendaal FR, Hammerstrom J. Incidence and mortality of venous thrombosis: a population-based study. J Thromb Haemost. 2007;5(4):692-9.

7. Van Beek E, Buller H, Ten Cate J. Epidemiology of venous thromboembolism. A textbook of vascular medicine. London: Arnold; 1996. p. 471-88.

8. Silverstein MD, Heit JA, Mohr DN, Petterson TM, O'Fallon WM, Melton LJ 3rd. Trends in the incidence of deep vein thrombosis and pulmonary embolism: a 25-year population-based study. Arch Intern Med. 1998;158(6):585-93.

9. Prandoni P, Lensing AW, Cogo A, et al. The longterm clinical course of acute deep venous thrombosis. Ann Intern Med. 1996;125(1):1-7.

10. Mohr DN, Silverstein MD, Heit JA, Petterson TM, O'fallon WM, Melton LJ. The venous stasis syndrome after deep venous thrombosis or pulmonary embolism: a population-based study. Mayo Clin Proc. 2000;75(12):1249-56.

11. Franzeck UK, Schalch I, Jäger KA, Schneider E, Grimm J, Bollinger A. Prospective 12-year follow-up study of clinical and hemodynamic sequelae after deep vein thrombosis in low-risk patients (Zürich study). Circulation. 1996;93(1):74-9.

12. Beyth RJ, Cohen AM, Landefeld CS. Long-term outcomes of deep-vein thrombosis. Arch Intern Med. 1995;155(10):1031-7.

13. Kahn S, Comerota A, Cushman M. Council on Clinical Cardiology, Council on Cardiovascular and Stroke Nursing. The postthrombotic syndrome: evidence-based prevention, diagnosis, and treatment strategies: a scientific statement from the American Heart Association. Circulation. 2014;130(18):1636-61.

14. Prandoni P, Frulla M, Sartor D, Concolato A, Girolami A. Vein abnormalities and the post-thrombotic syndrome. J Thromb Haemost. 2005;3(2):401-2.

15. Vedovetto V, Dalla Valle F, Milan M, Pesavento R, Prandoni P. Residual vein thrombosis and transpopliteal reflux in patients with and without the post-thrombotic syndrome. Thromb Haemost. 2013;110(4):854-5.

16. Galanaud J, Holcroft C, Rodger M, et al. Predictors of post-thrombotic syndrome in a population with a first deep vein thrombosis and no primary venous insufficiency. J Thromb Haemost. 2013;11(3):474-80.

17. Tick L, Kramer M, Rosendaal F, Faber W, Doggen C. Risk factors for post-thrombotic syndrome in patients with a first deep venous thrombosis. J Thromb Haemost. 2008;6(12):2075-81. 
18. Comerota AJ, Grewal N, Martinez JT, et al. Postthrombotic morbidity correlates with residual thrombus following catheter-directed thrombolysis for iliofemoral deep vein thrombosis. J Vasc Surg. 2012;55(3):768-73.

19. Budnik I, Brill A. Immune factors in deep vein thrombosis initiation. Trends Immunol. 2018;39:610-23.

20. Henke PK, Wakefield T. Thrombus resolution and vein wall injury: dependence on chemokines and leukocytes. Thromb Res. 2009;123:S72-8.

21. Rabinovich A, Cohen JM, Cushman M, et al. Inflammation markers and the risk of post thrombotic syndrome: results from the Bio-Sox Study. Blood. 2013;122(21):36.

22. Rabinovich A, Cohen JM, Cushman M, et al. Association between inflammation biomarkers, anatomic extent of deep venous thrombosis, and venous symptoms after deep venous thrombosis. J Vasc Surg Venous Lymphat Disord. 2015;3(4):347-53.

23. Roumen-Klappe E, Janssen M, Van Rossum J, et al. Inflammation in deep vein thrombosis and the development of post-thrombotic syndrome: a prospective study. J Thromb Haemost. 2009;7(4):582-7.

24. Shbaklo H, Holcroft CA, Kahn SR. Levels of inflammatory markers and the development of the post-thrombotic syndrome. J Thromb Haemost. 2009;101(03):505-12.

25. Bouman A, Smits J, Ten Cate H, Ten Cate-Hoek A. Markers of coagulation, fibrinolysis and inflammation in relation to post-thrombotic syndrome. J Thromb Haemost. 2012;10(8):1532-8.

26. Korthuis RJ, Gute DC. Adhesion molecule expression in postischemic microvascular dysfunction: activity of a micronized purified flavonoid fraction. J Vasc Res. 1999;36(Suppl 1):15-23.

27. Takase S, Lerond L, Bergan JJ, Schmid-Schonbein GW. The inflammatory reaction during venous hypertension in the rat. Microcirculation. 2000;7(1):41-52.

28. Takase S, Pascarella L, Lerond L, Bergan JJ, SchmidSchonbein GW. Venous hypertension, inflammation and valve remodeling. Eur J Vasc Endovasc Surg. 2004;28(5):484-93.

29. Maria das Graças C, Cyrino FZ, de Carvalho JJ, Blanc-Guillemaud V, Bouskela E. Protective effects of micronized purified flavonoid fraction (MPFF) on a novel experimental model of chronic venous hypertension. Eur J Vasc Endovasc Surg. 2018;55(5):694-702.

30. Shoab SS, Porter JB, Scurr JH, Coleridge-Smith PD. Effect of oral micronized purified flavonoid fraction treatment on leukocyte adhesion molecule expression in patients with chronic venous disease: a pilot study. J Vasc Surg. 2000;31(3):456-61.

31. Bogachev VY, Boldin BV, Lobanov VN. Benefits of micronized purified flavonoid fraction as adjuvant therapy on inflammatory response after sclerotherapy. Int Angiol. 2018;37(1):71-8.

32. Wells PS, Anderson DR, Rodger M, et al. Evaluation of D-dimer in the diagnosis of suspected deep-vein thrombosis. N Eng J Med. 2003;349(13):1227-35.

33. Vasquez MA, Munschauer CE. Venous Clinical Severity Score and quality-of-life assessment tools: application to vein practice. Phlebology. 2008:259-75.

34. CIVIQ users' guide. http://www.civiq-20.com. Accessed 19 Oct 2018.

35. Villalta S, Bagatella P, Piccioli A, Lensing A, Prins M, Prandoni P. Assessment of validity and reproducibility of a clinical scale for the post-thrombotic syndrome [abstract]. Haemostasis. 1994(24 suppl 1):158a.

36. Marder VJ, Soulen RL, Atichartakarn V, et al. Quantitative venographic assessment of deep vein thrombosis in the evaluation of streptokinase and heparin therapy. J Lab Clin Med. 1977;89(5):1018-29.

37. Schulman S, Kearon C. Definition of major bleeding in clinical investigations of antihemostatic medicinal products in non-surgical patients. J Thromb Haemost. 2005;3(4):692-4.

38. Henke PK, Wakefield TW, Diaz JA. Acute and chronic venous thrombosis: pathogenesis and new insights. Handbook of venous and lymphatic disorders. Boca Raton: CRC; 2017. p. 121-36.

39. Meissner MH, Caps MT, Bergelin RO, Manzo RA, Strandness DE Jr. Propagation, rethrombosis and new thrombus formation after acute deep venous thrombosis. J Vasc Surg. 1995;22(5):558-67.

40. Egermayer P. The effects of heparin and oral anticoagulants on thrombus propagation and prevention of the postphlebitic syndrome: a critical review of the literature. Prog Cardiovasc Dis. 2001;44(1):69-80.

41. Hull RD, Liang J, Townshend G. Long-term lowmolecular-weight heparin and the post-thrombotic 
syndrome: a systematic review. Am J Med. 2011;124(8):756-65.

42. Poredos P, Jezovnik M. Heparin promotes recanalization of venous thrombotic occlusions. Int Angiol. 2018;37(4):261-8.

43. Roussin A. Effective management of acute deep vein thrombosis: direct oral anticoagulants. Int Angiol. 2015;34(1):16-29.

44. Utne K, Dahm A, Wik H, Jelsness-Jørgensen L, Sandset P, Ghanima W. Rivaroxaban versus warfarin for the prevention of post-thrombotic syndrome. Thromb Res. 2018;163:6-11.

45. Jeraj L, Jezovnik M, Poredos P. Rivaroxaban versus warfarin in the prevention of post-thrombotic syndrome. Thromb Res. 2017;157:46-8.

46. Prandoni P, Ageno W, Mumoli N, et al. Recanalization rate in patients with proximal vein thrombosis treated with the direct oral anticoagulants. Thromb Res. 2017;153:97-100.

47. Rothmeier AS, Ruf W. Protease-activated receptor 2 signaling in inflammation. Semin Immunopathol. 2012;34(1):133-4.

48. Ramacciotti E, Volpiani GG, Davila R, Resende VA, Silveira FM, Fareed J. Early recanalization of veins in patients with DVT treated with rivaroxaban: do we need to move from bedside to bench again? Int Angiol. 2018;37(1):1-3.

49. Piovella F, Crippa L, Barone M, et al. Normalization rates of compression ultrasonography in patients with a first episode of deep vein thrombosis of the lower limbs: association with recurrence and new thrombosis. Haematologica. 2002;87(5):515-22.

50. Siragusa S, Malato A, Anastasio R, et al. Residual vein thrombosis to establish duration of anticoagulation after a first episode of deep vein thrombosis: the Duration of Anticoagulation based on Compression UltraSonography (DACUS) study. Blood. 2008;112(3):511-5

51. Prandoni $\mathrm{P}$, Lensing AW, Prins $\mathrm{MH}$, et al. Residual venous thrombosis as a predictive factor of recurrent venous thromboembolism. Ann Intern Med. 2002;137(12):955-60.

52. Prandoni P, Prins $M H$, Lensing AW, et al. Residual thrombosis on ultrasonography to guide the duration of anticoagulation in patients with deep venous thrombosis: a randomized trial. Ann Intern Med. 2009;150(9):577-85.

53. Trinh F, Paolini D, Fish J, Kasper G, Lurie F. Use of Villalta score for defining post-thrombotic disease may lead to false-positive diagnosis in $42 \%$ of patients with primary chronic venous disease. J Vasc Surg Venous Lymphat Disord. 2018;6(2):291.

54. Kakkos SK, Nicolaides AN. Efficacy of micronized purified flavonoid fraction (Daflon ${ }^{\circledR}$ ) on improving individual symptoms, signs and quality of life in patients with chronic venous disease: a systematic review and meta-analysis of randomized doubleblind placebo-controlled trials. Int Angiol. 2018;37(2):143-54. 